\title{
Variational Methods for a Fractional Dirichlet Problem Involving Jumarie's Derivative
}

\author{
Rafał Kamocki \\ Faculty of Mathematics and Computer Science, University of Lodz, Banacha 22, 90-238 Lodz, Poland \\ Correspondence should be addressed to Rafał Kamocki; rafkam@math.uni.lodz.pl
}

Received 27 April 2015; Accepted 8 June 2015

Academic Editor: Efstratios Tzirtzilakis

Copyright (C) 2015 Rafał Kamocki. This is an open access article distributed under the Creative Commons Attribution License, which permits unrestricted use, distribution, and reproduction in any medium, provided the original work is properly cited.

\begin{abstract}
We investigate a fractional Dirichlet problem involving Jumarie's derivative. Using some variational methods a theorem on the existence and uniqueness of a solution to such problem is proved. In the proof of the main result we use a fractional counterpart of the du Bois-Reymond fundamental lemma.
\end{abstract}

\section{Introduction}

In the last time, fractional calculus plays an essential role in the fields of mathematics, physics, electronics, mechanics, engineering, and so forth (cf. [1-5]). Many processes in physics and engineering can be described accurately by using systems of differential equations containing different type of fractional derivatives. Among definitions of derivatives of fractional order we can pick the Riemann-Liouville and the Caputo derivatives out. Unfortunately, each of them has different unusual properties. For instance, the RiemannLiouville derivative of a constant is not zero and the Caputo derivative is defined only for differentiable functions (alternatively, for such functions that have no first order derivative but then they might have fractional derivatives of all orders less than one, see [6]).

Recently, Jumarie proposed a new definition of the fractional derivative being a little modification of the RiemannLiouville derivative (cf. [7-10]). His definition eliminates disadvantages of mentioned earlier derivatives, because the Jumarie derivative of a constant is equal to zero and it is defined for any continuous (nondifferentiable) functions.

In the paper we consider the following fractional boundary problem:

$$
\begin{aligned}
\left(x^{(\alpha)}\right)^{(\alpha)}(t) & =F_{x}(t, x(t)), \quad \text { for a.e. } t \in[a, b], \\
x(a) & =x(b)=0,
\end{aligned}
$$

where $\alpha \in(1 / 2,1), F:[a, b] \times \mathbb{R}^{n} \rightarrow \mathbb{R}$, and $x^{(\alpha)}$ denotes Jumarie's derivative of a function $x$. The above problem is a generalization of the classical Dirichlet problem of the form

$$
\begin{aligned}
x^{\prime \prime}(t) & =F_{x}(t, x(t)), \\
x(a) & =x(b)=0 .
\end{aligned}
$$

We discuss the problem of the existence of solutions to above problem. In our investigations we use some variational method given in [11]. First, we consider some integral functional depending on the Jumarie derivative, for which (1) is the Euler-Lagrange equation. Next, we prove existence of a critical point of mentioned functional in an appropriate space of functions and under suitable assumptions of regularity, coercivity, and convexity. In order to do it, we use the following.

Proposition 1 (see [11]). If $X$ is a reflexive Banach space and the functional $\mathscr{L}: X \rightarrow \mathbb{R}$ is coercive and sequentially weakly lower semicontinuos, then it possesses at least one minimum at $x_{0} \in X$.

Let us remind that a functional $\mathscr{L}$ defined on a Banach space $X$ is coercive if $\mathscr{L}(x) \rightarrow \infty$ whenever $\|x\| \rightarrow \infty$, and $\mathscr{L}$ is sequentially weakly lower semicontinuous at $x_{0} \in X$ if $\liminf _{n \rightarrow \infty} \mathscr{L}\left(x_{n}\right) \geq \mathscr{L}\left(x_{0}\right)$ for any sequence $\left\{x_{n}\right\} \subset X$ such that $x_{n} \rightarrow x_{0}$ weakly in $X$. 
Of course such critical point is a minimizer of this functional and it generates the solution to problem (1)-(2). In order to prove that a minimum point gives a solution it is sufficient to apply a fractional version of the du BoisReymond lemma obtained in Section 3. Results of a such type for the Dirichlet problem involving the Riemann-Liouville derivative have been obtained in [12].

The paper is organized as follows. In Section 2, we review some basic definitions and facts concerning fractional calculus that we need in the sequel. Moreover, we introduce the space of solutions to considered problem and give some useful properties of this space. In Section 3, we formulate and prove some fractional version of the du Bois-Reymond Lemma, which we use in the proof of the main result. Mentioned main results of the work, namely, a theorem on the existence and uniqueness of a solution to problem (1)-(2), are formulated and proved in Section 4.

\section{Preliminaries}

In the first part of this paper we recall some basic facts concerning fractional calculus (cf. [3, 7-10, 13]). Next, we introduce some function spaces, which will be used later.

2.1. Fractional Calculus. We will assume that $[a, b] \subset \mathbb{R}$ is a bounded interval.

Let $\alpha>0$ and $f \in L^{1}\left([a, b], \mathbb{R}^{n}\right)$. The left-sided RiemannLiouville integral of the function $f$ of order $\alpha$ is defined by

$$
\left(I_{a+}^{\alpha} f\right)(t):=\frac{1}{\Gamma(\alpha)} \int_{a}^{t} \frac{f(\tau)}{(t-\tau)^{1-\alpha}} d \tau, \quad t \in[a, b] \text { a.e. }
$$

In the rest of this paper we will assume that $\alpha \in(0,1)$.

The left-sided Riemann-Liouville derivative $D_{a+}^{\alpha} f$ of the function $f$ of order $\alpha$ is defined in the following way:

$$
\left(D_{a+}^{\alpha} f\right)(t):=\frac{d}{d t}\left(I_{a+}^{1-\alpha} f\right)(t), \quad t \in[a, b] \text { a.e., }
$$

provided that $I_{a+}^{1-\alpha} f$ has an absolutely continuous representant on $[a, b]$ (i.e., there exists an absolutely continuous function on $[a, b]$ which is equal a.e. on $[a, b]$ to $\left.I_{a+}^{1-\alpha} f\right)$.

Now, let us assume that $f \in C\left([a, b], \mathbb{R}^{n}\right)$.

Jumarie's modified Riemann-Liouville derivative of the function $f$ of order $\alpha$ is defined by

$$
\begin{aligned}
& f^{(\alpha)}(t):=\left(D_{a+}^{\alpha}(f(\cdot)-f(a))\right)(t) \\
&= \frac{1}{\Gamma(1-\alpha)} \frac{d}{d t} \int_{a}^{t} \frac{f(\tau)-f(a)}{(t-\tau)^{\alpha}} d \tau \\
& \\
& \quad t \in[a, b] \text { a.e., }
\end{aligned}
$$

provided that $I_{a+}^{1-\alpha}(f(\cdot)-f(a))$ has an absolutely continuous representant on $[a, b]$.

Remark 2. It is easy to see that if $f(a)=0$, then defined above derivatives coincide. Moreover, Jumarie's modified RiemannLiouville derivative of a constant equals zero.
Remark 3. The definition of fractional derivative given by (6) is a consequence of the following fractional derivative via difference reads defined by Jumarie:

$$
f^{(\alpha)}(t):=\lim _{h \rightarrow 0} h^{-\alpha} \sum_{k=1}^{\infty}(-1)^{k}\left(\begin{array}{l}
\alpha \\
k
\end{array}\right) f[x+(\alpha-k) h] .
$$

The $(d t)^{\alpha}$ integral of $f$ is given by

$$
\int_{a}^{x} f(t)(d t)^{\alpha}:=\alpha \int_{a}^{x} f(t)(x-t)^{\alpha-1} d t, \quad x \in[a, b] .
$$

We have the following theorem on the integration by parts.

Theorem 4. Let $f, g \in C\left([a, b], \mathbb{R}^{n}\right)$ and there exist derivatives $f^{(\alpha)}$ and $g^{(\alpha)}$. Then

$$
\begin{aligned}
\int_{a}^{b} f^{(\alpha)}(t) g(t)(d t)^{\alpha} \\
=\Gamma(\alpha+1)(f(b) g(b)-f(a) g(a)) \\
\quad-\int_{a}^{b} f(t) g^{(\alpha)}(t)(d t)^{\alpha} .
\end{aligned}
$$

2.2. Space $L^{p, \alpha}\left([a, b], \mathbb{R}^{n}\right)$. Let $1 \leqslant p<\infty$.

Let us define the set

$$
\begin{aligned}
& L^{p, \alpha}\left([a, b], \mathbb{R}^{n}\right):=\{f:[a, b] \\
& \quad \longrightarrow \mathbb{R}^{n} ; f \text {-measurable, }(b-\cdot)^{(\alpha-1) / p} f \\
& \left.\quad \in L^{p}\left([a, b], \mathbb{R}^{n}\right)\right\}
\end{aligned}
$$

with the norm

$$
\begin{aligned}
\|f\|_{L^{p, \alpha}} & :=\left\|(b-\cdot)^{(\alpha-1) / p} f\right\|_{L^{p}} \\
& =\left(\int_{a}^{b}|f(t)|^{p}(b-t)^{\alpha-1} d t\right)^{1 / p} .
\end{aligned}
$$

We will identify two functions belonging to $L^{p, \alpha}\left([a, b], \mathbb{R}^{n}\right)$ that coincide a.e. on $[a, b]$.

It is easy to show that $L^{p, \alpha}\left([a, b], \mathbb{R}^{n}\right)$ is a Banach space. In particular, the space $L^{2, \alpha}\left([a, b], \mathbb{R}^{n}\right)$, equipped with the inner product

$$
\langle f, g\rangle_{L^{2, \alpha}}:=\int_{a}^{b} f(t) g(t)(b-t)^{\alpha-1} d t
$$

is a Hilbert space.

Now, we give some properties of the space $L^{p, \alpha}\left([a, b], \mathbb{R}^{n}\right)$.

Proposition 5. The injection

$$
L^{p, \alpha}\left([a, b], \mathbb{R}^{n}\right) \subset L^{p}\left([a, b], \mathbb{R}^{n}\right)
$$

is continuous. 
Mathematical Problems in Engineering

3

Proof. Let $f \in L^{p, \alpha}\left([a, b], \mathbb{R}^{n}\right)$. Then

$$
\begin{aligned}
& \int_{a}^{b}|f(t)|^{p} d t \\
& \quad=\int_{a}^{b}(b-t)^{1-\alpha}(b-t)^{\alpha-1}|f(t)|^{p} d t \\
& \quad \leqslant(b-a)^{1-\alpha} \int_{a}^{b}\left((b-t)^{(\alpha-1) / p}|f(t)|\right)^{p} d t .
\end{aligned}
$$

Consequently,

$$
\|f\|_{L^{p}} \leqslant(b-a)^{(1-\alpha) / p}\|f\|_{L^{p, \alpha}} .
$$

The proof is completed.

Proposition 6 (Hölder inequality). Let $f \in L^{p, \alpha}\left([a, b], \mathbb{R}^{n}\right)$, $g \in L^{q, \alpha}\left([a, b], \mathbb{R}^{n}\right)$, and $1 / p+1 / q=1$. Then $f g \in$ $L^{1, \alpha}\left([a, b], \mathbb{R}^{n}\right)$ and

$$
\begin{aligned}
& \int_{a}^{b}|f(t) g(t)|(b-t)^{\alpha-1} d t \\
& \leqslant\left(\int_{a}^{b}|f(t)|^{p}(b-t)^{\alpha-1} d t\right)^{1 / p} \\
& \cdot\left(\int_{a}^{b}|g(t)|^{q}(b-t)^{\alpha-1} d t\right)^{1 / q} .
\end{aligned}
$$

Proof. From the Hölder inequality for the space $L^{p}\left([a, b], \mathbb{R}^{n}\right)$ we obtain

$$
\begin{aligned}
& \int_{a}^{b}|f(t) g(t)|(b-t)^{\alpha-1} d t \\
& \quad=\int_{a}^{b}\left((b-t)^{(\alpha-1) / p}|f(t)|\right)\left((b-t)^{(\alpha-1) / q}|g(t)|\right) d t \\
& \quad \leqslant\left(\int_{a}^{b}|f(t)|^{p}(b-t)^{\alpha-1} d t\right)^{1 / p} \\
& \quad \cdot\left(\int_{a}^{b}|g(t)|^{q}(b-t)^{\alpha-1} d t\right)^{1 / q} .
\end{aligned}
$$

Lemma 7. The operator $I_{a+}^{\alpha}: L^{p, \alpha}\left([a, b], \mathbb{R}^{n}\right) \rightarrow L^{p, \alpha}([a, b]$, $\left.\mathbb{R}^{n}\right)$ is bounded, and it means there exists a constant $C_{p}>0$ such that

$$
\left\|I_{a+}^{\alpha} f\right\|_{L^{p, \alpha}} \leqslant C_{p}\|f\|_{L^{p, \alpha}}, \quad f \in L^{p, \alpha}\left([a, b], \mathbb{R}^{n}\right) .
$$

Proof. Using Fubini's Theorem and [14, Lemma 1], we obtain

$$
\begin{aligned}
& \left\|I_{a+}^{\alpha} f\right\|_{L^{p, \alpha}}^{p}=\int_{a}^{b}(b-t)^{\alpha-1}\left|\left(I_{a+}^{\alpha} f\right)(t)\right|^{p} d t \leqslant c_{0} \int_{a}^{b}(b \\
& -t)^{\alpha-1} I_{a+}^{\alpha}|f(t)|^{p} d t=\frac{c_{0}}{\Gamma(\alpha)} \int_{a}^{b}\left(\int_{a}^{t}(b-t)^{\alpha-1}\right. \\
& \left.\cdot(t-\tau)^{\alpha-1}|f(\tau)|^{p} d \tau\right) d t=\frac{c_{0}}{\Gamma(\alpha)} \int_{a}^{b}|f(\tau)|^{p} \\
& \cdot\left(\int_{\tau}^{b}(b-t)^{\alpha-1}(t-\tau)^{\alpha-1} d t\right) d \tau \\
& =\left\{\begin{array}{l}
t=b-s(b-\tau) \\
d t=-d s(b-\tau)
\end{array}\right\}=\frac{c_{0}}{\Gamma(\alpha)} \int_{a}^{b}|f(\tau)|^{p} \\
& \cdot\left(\int_{0}^{1} s^{\alpha-1}(b-\tau)^{\alpha-1}(1-s)^{\alpha-1}(b-\tau)^{\alpha-1}\right. \\
& \cdot(b-\tau) d s) d \tau=\frac{c_{0}}{\Gamma(\alpha)} \int_{a}^{b}(b-\tau)^{2 \alpha-1}|f(\tau)|^{p} \\
& \cdot\left(\int_{0}^{1} s^{\alpha-1}(1-s)^{\alpha-1} d s\right) d \tau=\frac{\beta(\alpha, \alpha) c_{0}}{\Gamma(\alpha)} \int_{a}^{b}(b \\
& -\tau)^{\alpha-1}(b-\tau)^{\alpha}|f(\tau)|^{p} d \tau \leqslant \frac{\beta(\alpha, \alpha) c_{0}(b-a)^{\alpha}}{\Gamma(\alpha)} \\
& \cdot \int_{a}^{b}(b-\tau)^{\alpha-1}|f(\tau)|^{p} d \tau \\
& =\frac{\beta(\alpha, \alpha) c_{0}(b-a)^{\alpha}}{\Gamma(\alpha)}\|f\|_{L^{p, \alpha}}^{p},
\end{aligned}
$$

where $c_{0}=\left((b-a)^{\alpha} / \Gamma(\alpha+1)\right)^{p-1}, \beta(z, u):=\int_{0}^{1} r^{z-1}(1-$ $r)^{u-1} d r, z, u>0$.

Since $\beta(z, u)=\Gamma(z) \Gamma(u) / \Gamma(z+u)$, it suffices to put $C_{p}=$ $(b-a)^{\alpha}\left(\Gamma(\alpha) / \Gamma(2 \alpha) \Gamma(\alpha+1)^{p-1}\right)^{1 / p}$.

The proof is completed.

2.3. Space $I_{a+}^{\alpha}\left(L^{p, \alpha}\right)$. Let us define the set $I_{a+}^{\alpha}\left(L^{p, \alpha}\left([a, b], \mathbb{R}^{n}\right)\right)$ (shortly $\left.I_{a+}^{\alpha}\left(L^{p, \alpha}\right)\right)$ as follows:

$$
\begin{aligned}
& I_{a+}^{\alpha}\left(L^{p, \alpha}\right):=\left\{f:[a, b] \longrightarrow \mathbb{R}^{n} ; f\right. \\
& \left.\quad=I_{a+}^{\alpha} \varphi \text {, ae. on }[a, b], \varphi \in L^{p, \alpha}\left([a, b], \mathbb{R}^{n}\right)\right\} .
\end{aligned}
$$

Functions belonging to $I_{a+}^{\alpha}\left(L^{p, \alpha}\right)$ and equal a.e. on $[a, b]$ are identified.

From Proposition 5, we immediately obtain the following.

Proposition 8. Consider

$$
I_{a+}^{\alpha}\left(L^{p, \alpha}\right) \subset I_{a+}^{\alpha}\left(L^{p}\right),
$$

where $I_{a+}^{\alpha}\left(L^{p}\right):=\left\{f:[a, b] \rightarrow \mathbb{R}^{n} ; f=I_{a+}^{\alpha} h\right.$, are. on $[a, b]$, $\left.h \in L^{p}\left([a, b], \mathbb{R}^{n}\right)\right\}$. 
From the above fact and [14, Proposition 2] it follows that if $f \in I_{a+}^{\alpha}\left(L^{p, \alpha}\right)$ then there exists the Riemann-Liouville derivative $D_{a+}^{\alpha} f$ almost everywhere on $[a, b]$.

Moreover, one can show that $I_{a+}^{\alpha}\left(L^{p, \alpha}\right)$ with the norm $\|\cdot\|_{I_{a+}^{\alpha}\left(L^{p, \alpha}\right)}: I_{a+}^{\alpha}\left(L^{p, \alpha}\right) \rightarrow[0, \infty)$ given by

$$
\|f\|_{I_{a+}^{\alpha}\left(L^{p, \alpha}\right)}:=\left\|D_{a+}^{\alpha} f\right\|_{L^{p, \alpha}}
$$

is complete and, consequently, is a Banach space. In particular, the space $I_{a+}^{\alpha}\left(L^{2, \alpha}\right)$, equipped with the inner product

$$
\begin{aligned}
& \langle f, g\rangle_{I_{a+}^{\alpha}\left(L^{2, \alpha}\right)} \\
& \quad:=\int_{a}^{b}\left(D_{a+}^{\alpha} f\right)(t)\left(D_{a+}^{\alpha} g\right)(t)(b-t)^{\alpha-1} d t,
\end{aligned}
$$

is a Hilbert space.

Remark 9. Let us note that in the case $p>1 / \alpha$, from Proposition 8 and [15, Property 4], it follows that

$$
I_{a+}^{\alpha}\left(L^{p, \alpha}\right) \subset I_{a+}^{\alpha}\left(L^{p}\right) \subset C_{0}\left([a, b], \mathbb{R}^{n}\right),
$$

where $C_{0}\left([a, b], \mathbb{R}^{n}\right):=\left\{u \in C\left([a, b], \mathbb{R}^{n}\right): u(a)=0\right\}$. Consequently, if $f \in I_{a+}^{\alpha}\left(L^{p, \alpha}\right)$ then $f$ possesses Jumarie's modified Riemann-Liouville derivative $f^{(\alpha)}$ and $f^{(\alpha)}=D_{a+}^{\alpha} f$. Of course, then

$$
\|f\|_{I_{a+}^{\alpha}\left(L^{p, \alpha}\right)}:=\left\|f^{(\alpha)}\right\|_{L^{p, \alpha}} .
$$

Remark 10. From Proposition 8, monography [3, Lemma 2.5(a)] and Remarks 2 and 9 it follows that if $f \in I_{a+}^{\alpha}\left(L^{p, \alpha}\right)$ with $p>1 / \alpha$ then

$$
f(t)=I_{a+}^{\alpha} f^{(\alpha)}(t), \quad t \in[a, b] \text { a.e. }
$$

From the above remark and Lemma 7, we immediately obtain the following.

Lemma 11 (fractional Poincaré Inequality). Let $p>1 / \alpha$. Then

$$
\begin{aligned}
& \left(\int_{a}^{b}|f(t)|^{p}(b-t)^{\alpha-1} d t\right)^{1 / p} \\
& \quad \leq C_{p}\left(\int_{a}^{b}\left|f^{(\alpha)}(t)\right|^{p}(b-t)^{\alpha-1} d t\right)^{1 / p}
\end{aligned}
$$

for $f \in I_{a+}^{\alpha}\left(L^{p, \alpha}\right)$, where $C_{p}>0$ is the constant from Lemma 7 .

\section{Du Bois-Reymond Lemma}

In this section, we will prove the du Bois-Reymond lemma for nondifferentiable functions.

We have the following.

Lemma 12 (du Bois-Reymond lemma). Let $\alpha \in(1 / 2,1), f \in$ $L^{2, \alpha}\left([a, b], \mathbb{R}^{n}\right)$, and

$$
\int_{a}^{b} f(t) h^{(\alpha)}(t)(b-t)^{\alpha-1} d t=0
$$

for any function $h \in I_{a+}^{\alpha}\left(L^{2, \alpha}\right)$ such that $h(b)=0$. Then there exists a constant $c \in \mathbb{R}^{n}$ such that $f(t)=c$ for a.e. $t \in[a, b]$ and, consequently, $f^{(\alpha)}(t)=0$ for a.e. $t \in[a, b]$.

Proof. First, let us note that from the Hölder inequality (cf. Proposition 6) it follows that the integral (28) is well-defined. Let $w(t)=c$ for $t \in[a, b]$, where $c=\left(\alpha /(b-a)^{\alpha}\right) \int_{a}^{b} f(t)(b-$ $t)^{\alpha-1} d t$. Then, for any function $h \in I_{a+}^{\alpha}\left(L^{2, \alpha}\right)$ such that $h(b)=$ 0 (in view of Remark 9 the condition $h(a)=0$ is satisfied also), from assumption (28) and Theorem 4, we obtain

$$
\begin{aligned}
0= & \int_{a}^{b} f(t) h^{(\alpha)}(t)(b-t)^{\alpha-1} d t-0 \\
= & \int_{a}^{b} f(t) h^{(\alpha)}(t)(b-t)^{\alpha-1} d t \\
& -\frac{1}{\alpha} \int_{a}^{b} w^{(\alpha)}(t) h(t)(d t)^{\alpha} \\
= & \int_{a}^{b} f(t) h^{(\alpha)}(t)(b-t)^{\alpha-1} d t \\
& -\frac{1}{\alpha} \int_{a}^{b} w(t) h^{(\alpha)}(t)(d t)^{\alpha} \\
& +\frac{\Gamma(\alpha+1)}{\alpha}(w(b) h(b)-w(a) h(a)) \\
= & \int_{a}^{b}(f(t)-w(t)) h^{(\alpha)}(t)(b-t)^{\alpha-1} d t .
\end{aligned}
$$

Thus,

$$
\begin{aligned}
& \int_{a}^{b}(f(t)-w(t)) h^{(\alpha)}(t)(b-t)^{\alpha-1} d t=0, \\
& \quad h \in I_{a+}^{\alpha}\left(L^{2, \alpha}\right), h(b)=0 .
\end{aligned}
$$

Let us consider the function $\widehat{h}(t)=\left(I_{a+}^{\alpha}(f-w)\right)(t)$ for a.e. $t \in[a, b]$. It is easy to see that $\widehat{h} \in I_{a+}^{\alpha}\left(L^{2, \alpha}\right)$ and, in view of Remark 9, $\widehat{h}(a)=0$. We will show that $\widehat{h}(b)=0$. Indeed, we have

$$
\begin{aligned}
\widehat{h}(b)= & \frac{1}{\Gamma(\alpha)} \int_{a}^{b}(f(t)-w(t))(b-t)^{\alpha-1} d t \\
= & \frac{1}{\Gamma(\alpha)} \int_{a}^{b} f(t)(b-t)^{\alpha-1} d t \\
& -\frac{c}{\Gamma(\alpha)} \int_{a}^{b}(b-t)^{\alpha-1} d t \\
= & \frac{1}{\Gamma(\alpha)} \int_{a}^{b} f(t)(b-t)^{\alpha-1} d t-\frac{c}{\Gamma(\alpha)} \frac{(b-a)^{\alpha}}{\alpha}=0 .
\end{aligned}
$$

Consequently, the function $\widehat{h}$ satisfies equality (30). So,

$$
\int_{a}^{b}(f(t)-w(t))^{2}(b-t)^{\alpha-1} d t=0 .
$$


It means that

$$
f(t)=w(t)=c, \quad t \in[a, b] \text { a.e. }
$$

The proof is completed.

Remark 13. In [16] result of such a type, but for Caputo derivative (for differentiable functions $h$ ), had been proved.

Using Lemma 12, we will prove the next lemma, which will play a key role in the next section. We have the following.

Lemma 14. Let $\alpha \in(1 / 2,1), f_{1} \in L^{2, \alpha}\left([a, b], \mathbb{R}^{n}\right), f_{2} \in$ $L^{2}\left([a, b], \mathbb{R}^{n}\right)$, and

$$
\int_{a}^{b}\left(f_{1}(t) h^{(\alpha)}(t)+f_{2}(t) h(t)\right)(b-t)^{\alpha-1} d t=0
$$

for any function $h \in I_{a+}^{\alpha}\left(L^{2, \alpha}\right)$ such that $h(b)=0$. Then,

$$
f_{1}(t)=\left(I_{a+}^{\alpha} f_{2}\right)(t)+c, \quad t \in[a, b] \text { a.e., }
$$

where $c \in \mathbb{R}^{n}$ is the constant from Lemma 12, and consequently

$$
f_{1}^{(\alpha)}(t)=f_{2}(t), \quad t \in[a, b] \text { a.e. }
$$

Proof. Using the Hölder inequality for spaces $L^{p}$ and $L^{p, \alpha}$, we check that integral (34) exists. Let us put $v=I_{a+}^{\alpha} f_{2}$. Then $v \in I_{a+}^{\alpha}\left(L^{2}\right)$ and $v^{(\alpha)}=f_{2}$. From Theorem 4 and assumption (34), we obtain

$$
\begin{aligned}
& 0= \alpha \int_{a}^{b}\left(f_{1}(t) h^{(\alpha)}(t)+f_{2}(t) h(t)\right)(b-t)^{\alpha-1} d t \\
&= \alpha \int_{a}^{b} f_{1}(t) h^{(\alpha)}(t)(b-t)^{\alpha-1} d t \\
&+\int_{a}^{b} v^{(\alpha)}(t) h(t)(d t)^{\alpha} \\
&= \alpha \int_{a}^{b} f_{1}(t) h^{(\alpha)}(t)(b-t)^{\alpha-1} d t \\
& \quad-\int_{a}^{b} v(t) h^{(\alpha)}(t)(d t)^{\alpha} \\
&+\Gamma(\alpha+1)(v(b) h(b)-v(a) h(a)) \\
&= \alpha \int_{a}^{b}\left(f_{1}(t)-v(t)\right) h^{(\alpha)}(t)(b-t)^{\alpha-1} d t, \\
& \quad h \in I_{a+}^{\alpha}\left(L^{2, \alpha}\right), h(b)=0 .
\end{aligned}
$$

Thus

$$
\begin{aligned}
& \int_{a}^{b}\left(f_{1}(t)-v(t)\right) h^{(\alpha)}(t)(b-t)^{\alpha-1} d t=0, \\
& \quad h \in I_{a+}^{\alpha}\left(L^{2, \alpha}\right), h(b)=0 .
\end{aligned}
$$

From Lemma 12 it follows that there exists a constant $c \in \mathbb{R}^{n}$ such that

$$
f_{1}(t)-v(t)=c, \quad t \in[a, b] \text { a.e. }
$$

Consequently,

$$
f_{1}(t)=v(t)+c=\left(I_{a+}^{\alpha} f_{2}\right)(t)+c, \quad t \in[a, b] \text { a.e. }
$$

It is well known that the function $I_{a+}^{\alpha} f_{2}$ possesses the leftsided Riemann-Liouville derivative and

$$
\left(D_{a+}^{\alpha} I_{a+}^{\alpha} f_{2}\right)(t)=f_{2}(t), \quad t \in[a, b] \text { a.e. }
$$

Since $\alpha \in(1 / 2,1)$, from [15, Property 4] it follows that the function $I_{a+}^{\alpha} f_{2}$ is continuous and $\left(I_{a+}^{\alpha} f_{2}\right)(a)=0$. Consequently, it possesses also the Jumarie modified RiemannLiouville derivative, wchich equals $f_{2}$. It means that the function $f_{1}$ has the Jumarie modified Riemann-Liouville derivative and (using the second part of Remark 2)

$$
f_{1}^{(\alpha)}(t)=f_{2}(t), \quad t \in[a, b] \text { a.e. }
$$

The proof is completed.

\section{Main Result}

Let us consider Dirichlet problem (1)-(2). By a solution to such problem we will mean a function $x \in I_{a+}^{\alpha}\left(L^{2, \alpha}\right)$, satysfying condition $x(b)=0$, such that $x^{(\alpha)} \in\left(I_{a+}^{\alpha}\left(L^{2}\right)+\right.$ $\{c ; c \in \mathbb{R}\}$ ).

Let us notice that since $\alpha \in(1 / 2,1)$, from Remark 9 it follows that $x$ and $x^{(\alpha)}$ are continuous and $x$ satisfies the initial condition $x(a)=0$.

In order to prove the existence of solutions to problem (1)-(2), we use variational methods.

Let us consider a functional $\mathscr{L}$ of the form

$$
\mathscr{L}(x):=\int_{a}^{b}\left(\frac{1}{2}\left|x^{(\alpha)}(t)\right|^{2}+F(t, x(t))\right)(b-t)^{\alpha-1} d t
$$

defined on the following space

$$
\begin{aligned}
K_{0}^{2, \alpha}\left([a, b], \mathbb{R}^{n}\right) & =K_{0}^{2, \alpha} \\
& =\left\{x \in I_{a+}^{\alpha}\left(L^{2, \alpha}\right): x(b)=0\right\} .
\end{aligned}
$$

We impose the following assumption on the function $F$ :

(A1) The function

$$
[a, b] \ni t \longmapsto F(t, x) \in \mathbb{R}
$$

is measurable on $[a, b]$ for any $x \in \mathbb{R}^{n}$ and the function

$$
\mathbb{R}^{n} \ni x \longmapsto F(t, x) \in \mathbb{R}
$$

is of class $C^{1}$ on $\mathbb{R}$ for a.e. $t \in[a, b]$.

(A2) There exist constants $c_{1}, c_{2} \geq 0$ and functions $\gamma_{1} \in$ $L^{1, \alpha}\left([a, b], \mathbb{R}^{+}\right)$and $\gamma_{2} \in L^{2}\left([a, b], \mathbb{R}^{+}\right)$such that

$$
\begin{gathered}
|F(t, x)| \leq c_{1}|x|+\gamma_{1}(t), \\
\left|F_{x}(t, x)\right| \leq c_{2}|x|+\gamma_{2}(t)
\end{gathered}
$$

for almost every $t \in[a, b]$ and all $x \in \mathbb{R}^{n}$. 
We say that $\mathscr{L}$ possesses the first variation $\delta \mathscr{L}(x, h)$ at the point $x \in K_{0}^{2, \alpha}$ in the direction $h \in K_{0}^{2, \alpha}$ (cf. [17]) if there exists a finite limit

$$
\delta \mathscr{L}(x, h)=\lim _{\lambda \rightarrow 0} \frac{\mathscr{L}(x+\lambda h)-\mathscr{L}(x)}{\lambda} .
$$

We will prove that, under assumptions (A1) and (A2), $\mathscr{L}$ possesses its minimum at a point $x_{0}$ which is a solution to (1).

To begin with, we will prove the following.

Theorem 15. Let us assume that conditions (A1)-(A2) are satisfied. Then the functional $\mathscr{L}$ is well-defined on $K_{0}^{2, \alpha}$ and possesses the first variation $\delta \mathscr{L}(x, h)$ at any point $x \in K_{0}^{2, \alpha}$ and in any direction $h \in K_{0}^{2, \alpha}$ given by

$$
\begin{aligned}
\delta \mathscr{L} & (x, h) \\
& =\int_{a}^{b}\left(\left(x^{(\alpha)}\right)(t)\left(h^{(\alpha)}\right)(t)+F_{x}(t, x(t)) h(t)\right) \\
& \cdot(b-t)^{\alpha-1} d t .
\end{aligned}
$$

Proof. The fact that $\mathscr{L}$ and $\delta \mathscr{L}$ are well-defined follows directly from (A1)-(A2) and the Hölder inequality (cf. Proposition 6). Let us fix $x \in K_{0}^{2, \alpha}$ and $h \in K_{0}^{2, \alpha}$ and write the functional $\mathscr{L}$ as

$$
\mathscr{L}=\mathscr{L}_{2} \circ \mathscr{L}_{1}
$$

where

$$
\begin{aligned}
\mathscr{L}_{1}: K_{0}^{2, \alpha} & \ni x(\cdot) \\
& \longrightarrow \frac{1}{2}\left|x^{(\alpha)}(\cdot)\right|^{2}+F(\cdot, x(\cdot)) \\
& \in L^{1, \alpha}([a, b], \mathbb{R}), \\
\mathscr{L}_{2}: L^{1, \alpha}([a, b], \mathbb{R}) & \ni y(\cdot) \longrightarrow \int_{a}^{b} y(t)(b-t)^{\alpha-1} d t \\
& \in \mathbb{R} .
\end{aligned}
$$

It is clear that $\mathscr{L}_{1}$ and $\mathscr{L}_{2}$ are well-defined and $\mathscr{L}_{2}$ is linear. Moreover,

$$
\begin{aligned}
\left|\mathscr{L}_{2}(y)\right| \leqslant \int_{a}^{b}|y(t)|(b-t)^{\alpha-1} d t & =\|y\|_{L^{1, \alpha}}, \\
y & \in L^{1, \alpha}([a, b], \mathbb{R}),
\end{aligned}
$$

so $\mathscr{L}_{2}$ is continuous. Consequently, it is differentiable in the sense of Frechet on $L^{1, \alpha}([a, b], \mathbb{R})$ and the differential at any point $y \in L^{1, \alpha}([a, b], \mathbb{R})$ is equal to $\mathscr{L}_{2}$. Using the Lebesque dominated convergence theorem and the mean value theorem, we assert that the mapping $\mathscr{L}_{1}$ has the first variation $\delta \mathscr{L}_{1}(x, h)$ at any point $x \in K_{0}^{2, \alpha}$ and in any direction $h \in K_{0}^{2, \alpha}$ given by

$$
\begin{aligned}
\delta \mathscr{L}_{1}(x(\cdot), h(\cdot))= & \left(x^{(\alpha)}\right)(\cdot)\left(h^{(\alpha)}\right)(\cdot) \\
& +F_{x}(\cdot, x(\cdot)) h(\cdot) .
\end{aligned}
$$

This means (cf. [17, Section 2.2.2]) that there exists the first variation of the mapping $\mathscr{L}$ given by equality (49).

The proof is completed.

Using the same arguments as in [15, Proposition 1 (3.3)], we can obtain the following.

Theorem 16. Let $\alpha \in(1 / 2,1)$ and $\left(f_{k}\right)_{k \in \mathbb{N}} \subset K_{0}^{2, \alpha}$. If $f_{k} \rightarrow$ $f$ weakly in $K_{0}^{2, \alpha}$ (with topology induced from $I_{a+}^{\alpha}\left(L^{2, \alpha}\right)$ ), then $f_{k} \rightrightarrows f$ uniformly on $[a, b]$.

Now, we will prove the main result of this paper, namely, a theorem on the existence of a unique solution to problem (1)-(2). We have the following.

Theorem 17. Let $\alpha \in(1 / 2,1)$ and assume that assumptions (A1)-(A2) are satisfied. If there are constants $a_{1}<\alpha \Gamma(2 \alpha) /(b-$ $a)^{2 \alpha}, b_{1}, b_{2} \geq 0$, such that

$$
\begin{aligned}
F(t, x) \geq-\frac{a_{1}}{2}|x|^{2}-b_{1}|x|-b_{2}, & \\
& t \in[a, b] \text { a.e., } x \in \mathbb{R}^{n}
\end{aligned}
$$

then problem (1)-(2) possesses at least one solution which minimizes functional $\mathscr{L}$. Moreover, if the function

$$
\mathbb{R}^{n} \ni x \longmapsto F(t, x)+\frac{a_{1}|x|^{2}}{2} \in \mathbb{R}
$$

is convex for a.e. $t \in[a, b]$, then the solution is unique.

Proof. Let $x \in K_{0}^{2, \alpha}$. Then from condition (54), Proposition 6, and Lemma 11 it follows that

$$
\begin{aligned}
\mathscr{L}(x)= & \frac{1}{2}\|x\|_{I_{a+}^{\alpha}\left(L^{2, \alpha}\right)}^{2}+\int_{a}^{b} F(t, x(t))(b-t)^{\alpha-1} d t \\
\geq & \frac{1}{2}\|x\|_{I_{a+}^{\alpha}\left(L^{2, \alpha}\right)}^{2}-\frac{a_{1}}{2}\|x\|_{L^{2, \alpha}}^{2} \\
& -b_{1} \sqrt{\frac{(b-a)^{\alpha}}{\alpha}}\|x\|_{L^{2, \alpha}}-b_{2} \frac{(b-a)^{\alpha}}{\alpha} \\
\geq & \frac{1-a_{1} C_{2}^{2}}{2}\|x\|_{I_{a+}^{\alpha}\left(L^{2, \alpha}\right)}^{2} \\
& -b_{1} C_{2} \sqrt{\frac{(b-a)^{\alpha}}{\alpha}}\|x\|_{I_{a+}^{\alpha}\left(L^{2, \alpha}\right)}-b_{2} \frac{(b-a)^{\alpha}}{\alpha}
\end{aligned}
$$

where $C_{2}=(b-a)^{\alpha} / \sqrt{\alpha \Gamma(2 \alpha)}$ is the constant from Lemma 7 .

Consequently, since $a_{1}<\alpha \Gamma(2 \alpha) /(b-a)^{2 \alpha}, 1-a_{1} C_{2}^{2}>0$; so $\mathscr{L}$ is coercive.

Now, Let $x_{k} \rightarrow x_{0}$ weakly in $K_{0}^{2, \alpha}$. From Theorem 16 it follows that $x_{k} \rightrightarrows x_{0}$, on $[a, b]$. Thus and from assumption (A2), using the dominated convergence theorem, we get that

$$
\lim _{k \rightarrow \infty} \int_{a}^{b} F\left(t, x_{k}(t)\right) d t=\int_{a}^{b} F\left(t, x_{0}(t)\right) d t .
$$


Mathematical Problems in Engineering

7

Moreover, $K_{0}^{2, \alpha}$ is a Hilbert space as the closed subspace of $I_{a+}^{\alpha}\left(L^{2, \alpha}\right)$. Consequently, the mapping $x \mapsto\|x\|_{I_{a+}^{\alpha}\left(L^{2, \alpha}\right)}$ is weakly lower semicontinuous, so

$$
\begin{aligned}
\liminf _{k \rightarrow \infty} \mathscr{L}\left(x_{k}\right)= & \liminf _{k \rightarrow \infty} \frac{1}{2}\left\|x_{k}\right\|_{I_{a+}^{\alpha}\left(L^{2, \alpha}\right)}^{2} \\
& +\lim _{k \rightarrow \infty} \int_{a}^{b} F\left(t, x_{k}(t)\right) d t \\
\geq & \mathscr{L}\left(x_{0}\right) .
\end{aligned}
$$

This means that the functional $\mathscr{L}$ is sequentially weakly lower semicontinuous and, by the virtue of Proposition 1, we conclude that it possesses minimum at the point $x_{0} \in K_{0}^{2, \alpha}$.

From Theorem 15 and Fermat lemma it follows that

$$
\begin{aligned}
& \int_{a}^{b}\left(x_{0}^{(\alpha)}(t) h^{(\alpha)}(t)+F_{x}\left(t, x_{0}(t)\right) h(t)\right)(b-t)^{\alpha-1} d t \\
& \quad=0
\end{aligned}
$$

for any $h \in K_{0}^{2, \alpha}$. Applying Lemma 14, we get

$$
\left(x^{(\alpha)}\right)^{(\alpha)}(t)=F_{x}(t, x(t)) \quad \text { for a.e. } t \in[a, b] .
$$

Since $x_{0} \in K_{0}^{2, \alpha}$, boundary conditions $x(a)=x(b)=0$ are satisfied.

The proof of the existence part is completed.

Now, we will show that, under assumption (55), the solution to problem (1)-(2) is unique. First, let us note that for $x, y \in K_{0}^{2, \alpha}, x \neq y$, and $\lambda \in(0,1)$ we have

$$
\begin{aligned}
& (1-\lambda) \int_{a}^{b}\left(\left|x^{(\alpha)}(t)\right|^{2}-a_{1}|x(t)|^{2}\right)(b-t)^{\alpha-1} d t \\
& +\lambda \int_{a}^{b}\left(\left|y^{(\alpha)}(t)\right|^{2}-a_{1}|y(t)|^{2}\right)(b-t)^{\alpha-1} d t \\
& -\int_{a}^{b}\left(\left|(1-\lambda) x^{(\alpha)}(t)+\lambda y^{(\alpha)}(t)\right|^{2}\right. \\
& \left.-a_{1}|(1-\lambda) x(t)+\lambda y(t)|^{2}\right)(b-t)^{\alpha-1} d t=(1 \\
& -\lambda) \int_{a}^{b}\left(\left|x^{(\alpha)}(t)\right|^{2}-a_{1}|x(t)|^{2}\right)(b-t)^{\alpha-1} d t \\
& +\lambda \int_{a}^{b}\left(\left|y^{(\alpha)}(t)\right|^{2}-a_{1}|y(t)|^{2}\right)(b-t)^{\alpha-1} d t \\
& -\int_{a}^{b}\left((1-\lambda)^{2}\left|x^{(\alpha)}(t)\right|^{2}\right. \\
& \left.+2(1-\lambda) \lambda\left\langle x^{(\alpha)}(t), y^{(\alpha)}(t)\right\rangle+\lambda^{2}\left|y^{(\alpha)}(t)\right|^{2}\right)(b
\end{aligned}
$$

$$
\begin{aligned}
& -t)^{\alpha-1} d t+\int_{a}^{b}\left(a_{1}(1-\lambda)^{2}|x(t)|^{2}\right. \\
& \left.+2 a_{1}(1-\lambda) \lambda\langle x(t), y(t)\rangle+a_{1} \lambda^{2}|y(t)|^{2}\right)(b \\
& -t)^{\alpha-1} d t=\int_{a}^{b}\left((1-\lambda)\left|x^{(\alpha)}(t)\right|^{2}\right. \\
& -(1-\lambda)^{2}\left|x^{(\alpha)}(t)\right|^{2} \\
& \left.-2(1-\lambda) \lambda\left\langle x^{(\alpha)}(t), y^{(\alpha)}(t)\right\rangle\right)(b-t)^{\alpha-1} d t \\
& +\int_{a}^{b}\left(\lambda\left|y^{(\alpha)}(t)\right|^{2}-\lambda^{2}\left|y^{(\alpha)}(t)\right|^{2}\right)(b-t)^{\alpha-1} d t \\
& +\int_{a}^{b}\left(-a_{1}(1-\lambda)|x(t)|^{2}+a_{1}(1-\lambda)^{2}|x(t)|^{2}\right)(b \\
& -t)^{\alpha-1} d t+\int_{a}^{b}\left(2 a_{1}(1-\lambda) \lambda\langle x(t), y(t)\rangle\right. \\
& \left.-a_{1} \lambda|y(t)|^{2}+a_{1} \lambda^{2}|y(t)|^{2}\right)(b-t)^{\alpha-1} d t \\
& =\int_{a}^{b}\left((1-\lambda) \lambda\left|x^{(\alpha)}(t)\right|^{2}\right. \\
& -2(1-\lambda) \lambda\left\langle x^{(\alpha)}(t), y^{(\alpha)}(t)\right\rangle \\
& \left.+(1-\lambda) \lambda\left|y^{(\alpha)}(t)\right|^{2}\right)(b-t)^{\alpha-1} d t \\
& -a_{1} \int_{a}^{b}\left((1-\lambda) \lambda|x(t)|^{2}\right. \\
& \left.-2(1-\lambda) \lambda\langle x(t), y(t)\rangle+(1-\lambda) \lambda|y(t)|^{2}\right)(b \\
& -t)^{\alpha-1} d t=(1-\lambda) \\
& \cdot \lambda\left(\int_{a}^{b}\left|x^{(\alpha)}(t)-y^{(\alpha)}(t)\right|^{2}(b-t)^{\alpha-1} d t\right. \\
& \left.-a_{1} \int_{a}^{b}|x(t)-y(t)|^{2}(b-t)^{\alpha-1} d t\right) .
\end{aligned}
$$

If $a_{1} \leq 0$ then

$$
\begin{gathered}
(1-\lambda) \lambda\left(\int_{a}^{b}\left|x^{(\alpha)}(t)-y^{(\alpha)}(t)\right|^{2}(b-t)^{\alpha-1} d t\right. \\
\left.-a_{1} \int_{a}^{b}|x(t)-y(t)|^{2}(b-t)^{\alpha-1} d t\right) \geq(1-\lambda) \\
\cdot \lambda \int_{a}^{b}\left|x^{(\alpha)}(t)-y^{(\alpha)}(t)\right|^{2}(b-t)^{\alpha-1} d t>0,
\end{gathered}
$$


otherwise, from Lemma 11 and assumption $a_{1}<\alpha \Gamma(2 \alpha) /(b-$ a) ${ }^{2 \alpha}$, it follows that

$$
\begin{aligned}
& (1-\lambda) \lambda\left(\int_{a}^{b}\left|x^{(\alpha)}(t)-y^{(\alpha)}(t)\right|^{2}(b-t)^{\alpha-1} d t\right. \\
& \left.-a_{1} \int_{a}^{b}|x(t)-y(t)|^{2}(b-t)^{\alpha-1} d t\right) \geq(1-\lambda) \\
& \cdot \lambda\left(\int_{a}^{b}\left|x^{(\alpha)}(t)-y^{(\alpha)}(t)\right|^{2}(b-t)^{\alpha-1} d t\right. \\
& \left.-a_{1} \frac{(b-a)^{2 \alpha}}{\alpha \Gamma(2 \alpha)} \int_{a}^{b}\left|x^{(\alpha)}(t)-y^{(\alpha)}(t)\right|^{2}(b-t)^{\alpha-1} d t\right) \\
& =(1-\lambda) \lambda\left(1-a_{1} \frac{(b-a)^{2 \alpha}}{\alpha \Gamma(2 \alpha)}\right) \\
& \cdot \int_{a}^{b}\left|x^{(\alpha)}(t)-y^{(\alpha)}(t)\right|^{2}(b-t)^{\alpha-1} d t>0 .
\end{aligned}
$$

It means that the functional

$$
\begin{aligned}
K_{0}^{2, \alpha} & \ni x \\
& \longmapsto \frac{1}{2} \int_{a}^{b}\left(\left|x^{(\alpha)}(t)\right|^{2}-a_{1}|x(t)|^{2}\right)(b-t)^{\alpha-1} d t \\
& \in \mathbb{R}
\end{aligned}
$$

is strictly convex. Consequently, using assumption (55), we assert that the functional

$$
\begin{aligned}
\mathscr{L}(x)= & \int_{a}^{b}\left(F(t, x(t))+\frac{a_{1}}{2}|x(t)|^{2}\right)(b-t)^{\alpha-1} d t \\
& +\frac{1}{2} \int_{a}^{b}\left(\left|x^{(\alpha)}(t)\right|^{2}-a_{1}|x(t)|^{2}\right)(b-t)^{\alpha-1} d t
\end{aligned}
$$

is strictly convex, so its minimum point $x_{0} \in K_{0}^{2, \alpha}$ is unique. On the other hand if $x \in K_{0}^{2, \alpha}$ is a solution to (1)-(2) then

$$
\left(x_{0}^{(\alpha)}\right)^{(\alpha)}(t)=F_{x}\left(t, x_{0}(t)\right), \quad t \in[a, b] \text { a.e. }
$$

Thus,

$$
\begin{aligned}
& \int_{a}^{b}\left(x_{0}^{(\alpha)}\right)^{(\alpha)}(t) h(t)(b-t)^{\alpha-1} d t \\
& \quad-\int_{a}^{b} F_{x}\left(t, x_{0}(t)\right) h(t)(b-t)^{\alpha-1} d t=0
\end{aligned}
$$

for any $h \in K_{0}^{2, \alpha}$ and from Theorem 4 we get

$$
\begin{aligned}
& \int_{a}^{b} x_{0}^{(\alpha)}(t) h^{(\alpha)}(t)(b-t)^{\alpha-1} d t \\
& \quad+\int_{a}^{b} F_{x}\left(t, x_{0}(t)\right) h(t)(b-t)^{\alpha-1} d t=\delta \mathscr{L}\left(x_{0}, h\right) \\
& \quad=0 .
\end{aligned}
$$

This means that the solution $x_{0}$ to problem (1)-(2) is a minimum point of $\mathscr{L}$, so it is unique. The proof is completed.

\section{Conflict of Interests}

The author declares that there is no conflict of interests regarding the publication of this paper.

\section{Acknowledgment}

The project was financially supported by the Faculty of Mathematics and Computer Science, University of Lodz, under Grant no. B1411600000451.02 for young researchers and participants of a grad school.

\section{References}

[1] A. Carpinteri and F. Mainardi, Fractals and Fractional Calculus in Continuum Mechanics, Springer, Berlin, Germany, 1997.

[2] R. Hilfer, Applications of Fractional Calculus in Physics, World Scientific Publishing, River Edge, NJ, USA, 2000.

[3] A. A. Kilbas, H. M. Srivastava, and J. J. Trujillo, Theory and Applications of Fractional Differential Equations, Elsevier, Amsterdam, The Netherlands, 2006.

[4] S. G. Samko, A. A. Kilbas, and O. I. Marichev, Fractional Integrals and Derivatives and Some Their Applications, Gordon and Breach Science Publishers, Yverdon, Switzerland, 1993.

[5] B. J. West and P. Grigolini, Applications of Fractional Calculus in Physics, World Scientific, Singapore, 1998.

[6] B. Ross, S. G. Samko, and E. R. Love, "Functions that have no first order derivative might have fractional derivatives of all orders less than one," Real Analysis Exchange, vol. 20, no. 1, pp. 140-157, 1994-1995.

[7] G. Jumarie, "On the representation of fractional Brownian motion as an integral with respect to $(\mathrm{d} t)^{a}$," Applied Mathematics Letters, vol. 18, no. 7, pp. 739-748, 2005.

[8] G. Jumarie, "Stock exchange fractional dynamics defined as fractional exponential growth driven by (usual) Gaussian white noise. Application to fractional Black-Scholes equations," Insurance: Mathematics \& Economics, vol. 42, no. 1, pp. 271-287, 2008.

[9] G. Jumarie, "Table of some basic fractional calculus formulae derived from a modified Riemann-Liouville derivative for nondifferentiable functions," Applied Mathematics Letters, vol. 22, no. 3, pp. 378-385, 2009.

[10] G. Jumarie, Fractional Differential Calculus for Nondifferentiable Functions, LAP Lambert Academic Publishing, 2013.

[11] J. Mawhin, Problemes de Dirichlet Variationnels Non-Linéaires, vol. 104, Les Presses de L'Université de Montréal, Montreal, Canada, 1987.

[12] R. Kamocki and M. Majewski, "On a fractional Dirichlet problem," in Proceedings of the 17th International Conference on Methods and Models in Automation \& Robotics (MMAR '12), pp. 60-63, Miedzyzdrojie, Poland, August 2012.

[13] A. B. Malinowska and D. F. M. Torres, Introduction to the Fractional Calculus of Variations, Imperial College Press, London, UK, 2012.

[14] R. Kamocki, "Pontryagin maximum principle for fractional ordinary optimal control problems," Mathematical Methods in the Applied Sciences, vol. 37, no. 11, pp. 1668-1686, 2014.

[15] L. Bourdin, "Existence of a weak solution for fractional EulerLagrange equations," Journal of Mathematical Analysis and Applications, vol. 399, no. 1, pp. 239-251, 2013. 
[16] M. J. Lazo and D. F. Torres, "The DuBois-Reymond fundamental lemma of the fractional calculus of variations and an Euler-Lagrange equation involving only derivatives of Caputo," Journal of Optimization Theory and Applications, vol. 156, no. 1, pp. 56-67, 2013.

[17] V. M. Alekse'ev, V. M. Tikhomirov, and S. V. Fomin, Optimal Control, Fizmatlit, Moscow, Russia, 2005, (Russian). 


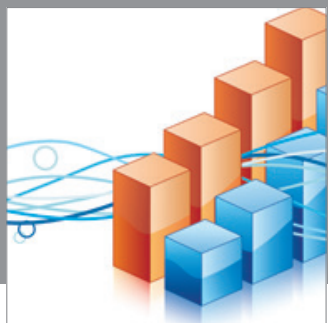

Advances in

Operations Research

mansans

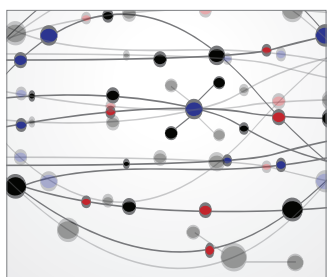

The Scientific World Journal
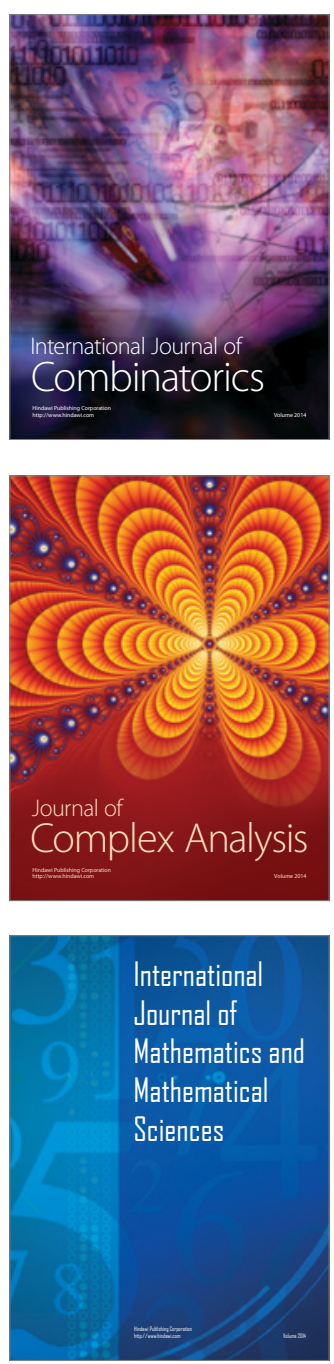
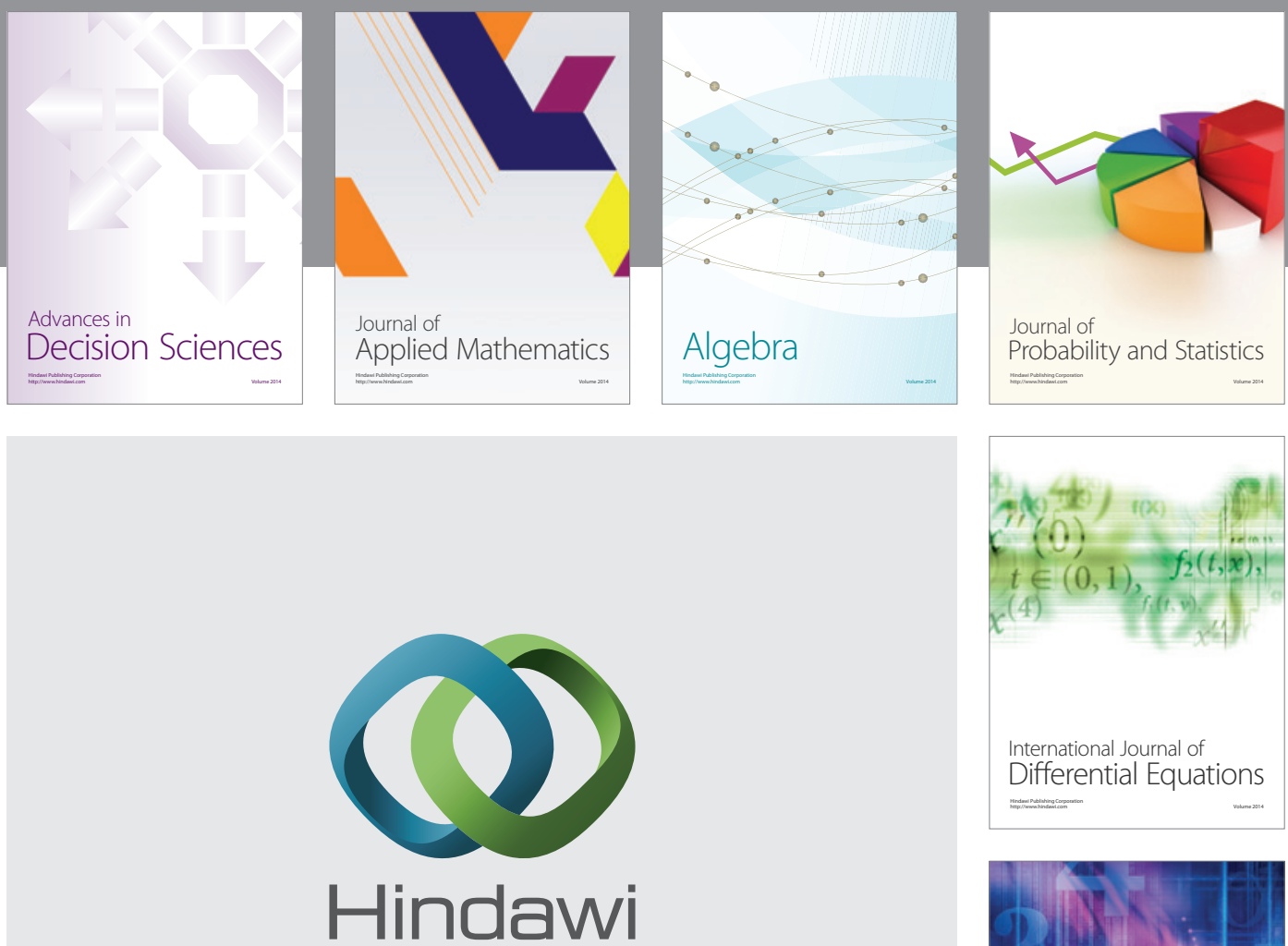

Submit your manuscripts at http://www.hindawi.com
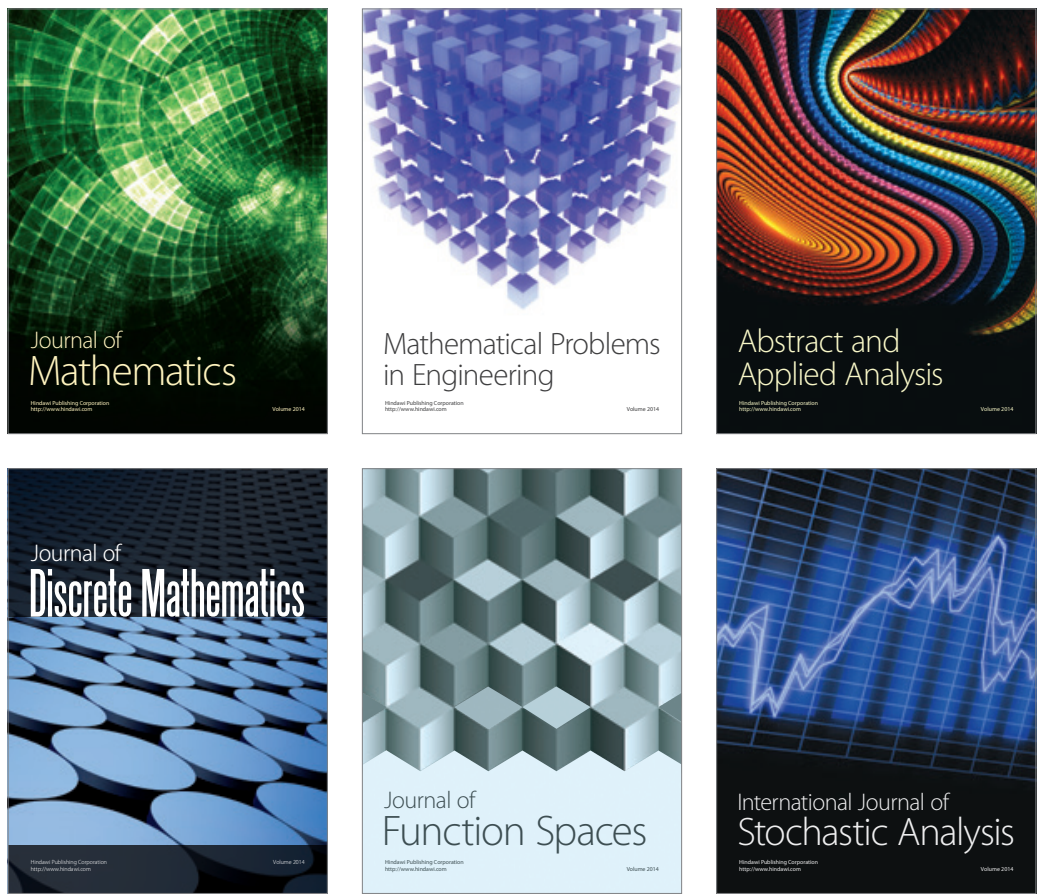

Journal of

Function Spaces

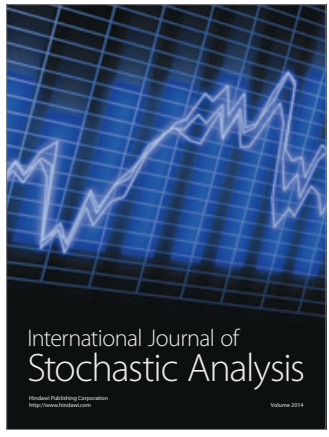

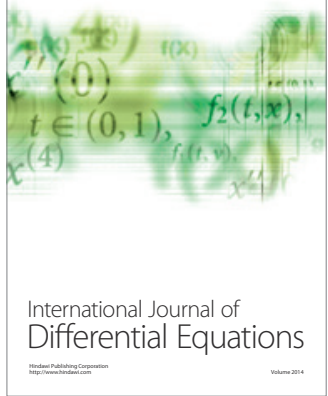
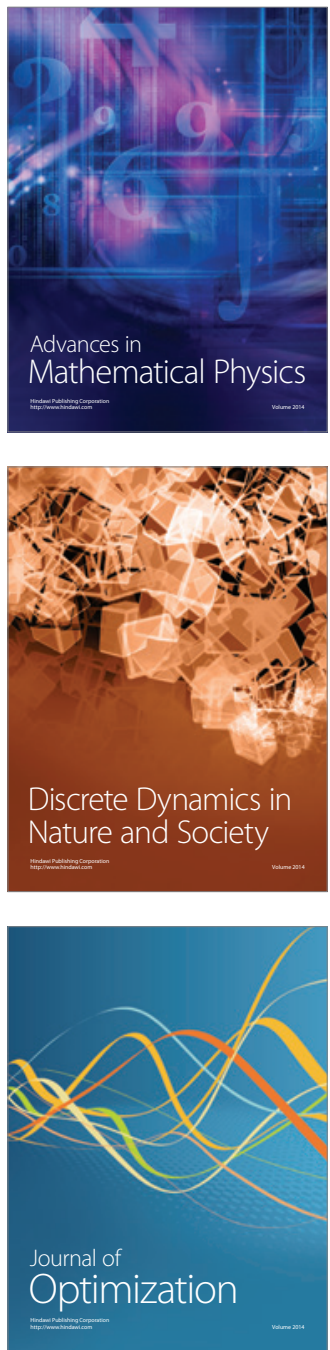